\title{
Use of dietary exposure modelling to estimate the intake of artificial sweeteners by young children with medical conditions
}

\author{
A. J. O'Sullivan ${ }^{1}$, M. J. Gibney ${ }^{2}$ and A. I. McKevitt ${ }^{2}$ \\ ${ }^{1}$ School of Biomedical Sciences, Faculty of Life and Health Sciences, University of Ulster, Coleraine, BT52 1SA and \\ ${ }^{2}$ School of Agriculture, Food Science and Veterinary Medicine, Agriculture and Food Science Centre \\ University College Dublin, Belfield, Dublin 4, Ireland
}

The range of foods available to individuals with particular medical conditions e.g. inborn errors of metabolism (Phenylketonuria, PKU) or severe cow's milk protein allergy (CMPA) is so limited that they must rely on specially formulated products to meet their nutrition requirements. Patients with PKU or CMPA have dietary restrictions along with exclusive or partial replacement with special formulations. Artificial sweeteners are used to improve the appearance and palatability of these formulations. Young children have a higher risk of exceeding acceptable daily intakes (ADI) for additives than adults due to higher food intakes per kg body weight ${ }^{(1,2,3)}$.

The aim was to use dietary exposure modelling to estimate the intake of artificial sweeteners by young PKU and CMPA patients (1-3 years). To date, none of the exposure models available have addressed additive intakes in young patients. EFSA's recently launched Food Additives Intake Model is not equipped to assess partial dietary replacement with special formulations. A new approach is needed.

Food consumption data for patients are not readily available. Data used for analysis are derived from the food consumption survey data of healthy young children as a baseline. The National Preschool and Nutrition Survey (NPNS) was designed to assess the diet, nutrient intake and nutritional status of the healthy Irish children aged 1-4 years. The population of interest was young children aged from 1-3 years (376 subjects). The food groups used for data collection in this study are the 17 EU food categories. An adapted validated probabilistic model was used to assess patients' exposure to artificial sweeteners ${ }^{(4,5,6)}$. Specially formulated products were included in the exposure model to replace restricted foods. Inclusion was based on recommendations for adequate protein intake and dietary adherence data.

The exposure assessment results indicate young Irish patients with PKU and CMPA have higher relative average intakes of artificial sweeteners than healthy young children (see tables).

Table 1. Additive exposure estimates for young patients with PKU

\begin{tabular}{|c|c|c|c|c|c|c|c|c|}
\hline & \multicolumn{8}{|l|}{ PKU } \\
\hline & \multicolumn{4}{|c|}{ Baseline $(\mathrm{mg} / \mathrm{kg} / \mathrm{d})$} & \multicolumn{4}{|c|}{ Patient model $(\mathrm{mg} / \mathrm{kg} / \mathrm{d})$} \\
\hline & Mean & $\mathrm{SE}$ & P95 & $\mathrm{SE}$ & Mean & $\mathrm{SE}$ & P95 & $\mathrm{SE}$ \\
\hline Sucralose & $3 \cdot 3$ & $0 \cdot 3$ & $16 \cdot 7$ & $1 \cdot 4$ & $9 \cdot 0$ & $0 \cdot 3$ & $22 \cdot 2$ & $1 \cdot 2$ \\
\hline AcesulfameK & $2 \cdot 2$ & $0 \cdot 2$ & $13 \cdot 4$ & $1 \cdot 1$ & $14 \cdot 2$ & $0 \cdot 2$ & $29 \cdot 5$ & $1 \cdot 1$ \\
\hline Aspartame & - & - & - & - & - & - & - & - \\
\hline
\end{tabular}

Table 2. Additive exposure estimates for young patients with CMPA

\begin{tabular}{|c|c|c|c|c|c|c|c|c|}
\hline & \multicolumn{8}{|c|}{ CMPA } \\
\hline & \multicolumn{4}{|c|}{ Baseline (mg/kg/d) } & \multicolumn{4}{|c|}{ Patient model $(\mathrm{mg} / \mathrm{kg} / \mathrm{d})$} \\
\hline & Mean & SE & P95 & $\overline{\mathrm{SE}}$ & Mean & SE & P95 & $\overline{\mathrm{SE}}$ \\
\hline Sucralose & $2 \cdot 4$ & $0 \cdot 3$ & $15 \cdot 7$ & $1 \cdot 0$ & $3 \cdot 3$ & $0 \cdot 3$ & $16 \cdot 6$ & $0 \cdot 3$ \\
\hline AcesulfameK & - & - & - & - & - & - & - & - \\
\hline Aspartame & $5 \cdot 2$ & $0 \cdot 6$ & $35 \cdot 7$ & $3 \cdot 1$ & $5 \cdot 5$ & $0 \cdot 6$ & $36 \cdot 1$ & $1 \cdot 4$ \\
\hline
\end{tabular}

Dietary exposure modelling provides a means of estimating dietary exposure to additives in patients which would otherwise not be possible. Based on modelled exposures certain high consumers (P95) with PKU may exceed the established ADI for Sucralose (15 mg/kg/day) and Acesulfame K (9 mg/kg/day). For CMPA certain high consumers (P95) may exceed the established ADI for Sucralose but not for Aspartame ( $40 \mathrm{mg} / \mathrm{kg} /$ day). Use of the EFSA FAIM model to estimate exposures would indicate that mean exposures of patients could exceed established ADIs for artificial sweeteners. The reliability and robustness of the model warrants further investigation in the estimation of patient additive exposures.

Work supported by Danone Trading B.V. Schiphol Boulevard 105, 1118 BG Schiphol Airport, The Netherlands

1. Bearer CF (1995) Future Child 5, 11-26.

2. Lawrie C (1998) Food Add Contam 15, 75-81.

3. VWA (2008) Report of a VWA panel. The Hague, The Netherlands.

4. McNamara C, Naddy B, Rohan D, Sexton J (2003) Food AdditContam. 20, S8-S26.

5. Gilsenan MB, Lambe J, Gibney MJ 2002. Food AdditContam. 19, 1105-1115.

6. Lambe J (2002) ProcNutr Soc. 61, 11-18. 